\title{
Estudio longitudinal sobre el tratamiento de la homeopatía en la prensa digital española (2012-2016)
}

\section{A longitudinal study about the treatment of homeopathy in the Spanish digital daily (2012-2016)}

\author{
Vanessa Roger-Monzó; Myriam Martí-Sánchez
}

Cómo citar este artículo:

Roger-Monzó, Vanessa; Martí-Sánchez, Myriam (2019). “Estudio longitudinal sobre el tratamiento de la homeopatía en la prensa digital española (2012-2016)”. El profesional de la información, v. 28, n. 2, e280216. https://doi.org/10.3145/epi.2019.mar.16

Artículo recibido el 04-08-2018 Aceptación definitiva: 22-03-2019

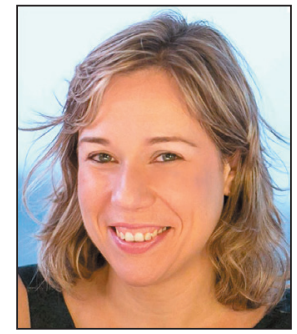

\section{Vanessa Roger-Monzó https://orcid.org/0000-0002-7498-0406}

ESIC Business \& Marketing School Avda. Blasco Ibáñez, 55. 46021 Valencia, España

vanessa.roger@esic.edu

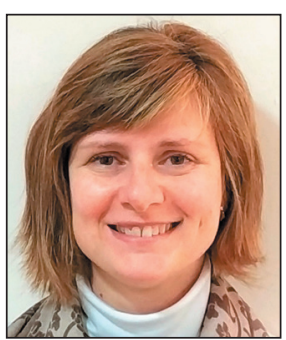

\section{Myriam Martí-Sánchez}

https://orcid.org/0000-0003-4344-4464

ESIC Business \& Marketing School Avda. Blasco Ibáñez, 55. 46021 Valencia, España

myriam.marti@esic.edu

\section{Resumen}

El objetivo de este estudio es evaluar cómo la prensa digital española aborda las informaciones sobre homeopatía. Para llevar a cabo la investigación, se ha realizado un análisis de contenido de los artículos publicados sobre homeopatía entre 1 de enero de 2012 y 31 de diciembre de 2016 en los tres diarios digitales de información general y ámbito nacional más leídos: elmundo.es, elpais.com y abc.es. La búsqueda ha recuperado 115 artículos. Los resultados revelan que la noticia es el género informativo más empleado, prevaleciendo el enfoque de controversia y conflicto como encuadre principal. Se antepone el valor noticia de impacto frente al análisis del fenómeno y de sus riesgos para la salud.

\section{Palabras clave}

Información de salud; Comunicación de salud; Prensa digital; Diarios; Periodismo especializado; Pseudociencias; Homeopatía; Agenda setting; Análisis de contenido.

\begin{abstract}
The aim of this study is to evaluate how the Spanish digital press deals with information related to homeopathy. To carry out the research, a content analysis of the stories published on homeopathy was developed between January $1^{\text {st }}, 2012$ and December 31 ${ }^{\text {st }}, 2016$ in the three most read digital Spanish newspapers: elmundo.es, elpais.com and abc.es. The search retrieved 115 articles. The results reveal that the piece of news is the most used informative genre, prevailing the focus of controversy and conflict as the main framework. The informative value of the impact is placed before the analysis of the phenomenon and its health risks.
\end{abstract}

Financiación

El presente trabajo ha sido desarrollado en el marco del proyecto de investigación Estudio y clasificación de las terapias naturales, complementarias y alternativas a través de los medios de comunicación y de las redes sociales. Ideas y valores de transferencia al imaginario social (CSO2014-57778-R), financiado por el Ministerio de Economía y Competitividad y por fondos Feder de la Unión Europea. 


\section{Keywords}

Health information; Health communication; Digital press; Newspapers; Specialized journalism; Pseudoscience; Homeopathy; Agenda setting; Content analysis.

\section{Introducción}

El Ministerio de Sanidad de España en su informe sobre las terapias alternativas incluyó 139 tipos de prácticas, entre ellas la homeopatía, determinando que la evidencia científica disponible sobre su eficacia es muy escasa. En el caso concreto de esta práctica,

"no ha probado definitivamente su eficacia en ninguna indicación o situación clínica concreta y los resultados de los ensayos clínicos disponibles son muy contradictorios" (Ministerio de Sanidad, 2011).

Aunque la homeopatía carece de evidencias científicas, la VIII Encuesta de percepción social de la ciencia y la tecnología realizada por la Fundación Española para la Ciencia y la Tecnología (Fecyt, 2017), dependiente del Ministerio de Economía, Industria y Competitividad, revela que el $50 \%$ de los participantes considera que es muy efectiva o algo efectiva. También se señala que esta percepción constituye un riesgo para la población, ya que se trata de una disciplina pseudocientífica cuyo uso se está incrementando durante los últimos años.

En esta misma línea, el Barómetro de febrero de 2018 del Centro de Investigaciones Sociológicas (CIS, 2018) incluye por primera vez diversas cuestiones en torno a la homeopatía. El resultado muestra un gran desconocimiento por parte de la sociedad española sobre la diferencia entre los tratamientos médicos y falsos remedios, si bien los datos también revelan un consumo mucho menor de lo que se estimaba hasta el momento (5\%).

\subsection{La homeopatía en los medios de comunicación}

La homeopatía se remonta al siglo XVIII y consiste en administrar dosis muy bajas de las sustancias que pueden generar una afección similar a la que se quiere combatir (Benkemoun et al., 2002). Esta práctica se basa en un sistema de creencias orientado a la prevención, curación o alivio de dolencias que carece de certezas científicas que demuestren su eficacia más allá del efecto placebo (Mathie et al., 2017; Rutten; Stolper, 2009; Ernst, 2008).

Etimológicamente el término pseudociencia equivale a "falsa ciencia" e intenta mostrar experiencias en las que se crea la impresión de solidez científica (Cornejo-Valle; Blázquez-Rodríguez, 2013), sin utilizar métodos exhaustivos y rigurosos en sus investigaciones (Leaf et al., 2016; Bracanović, 2012; Kurtz, 1978; 1981). La pseudociencia no tolera la crítica o el debate (Ernst, 2009) y su discurso se fundamenta en la utilización de un lenguaje emotivo y directo para hacer pasar las creencias como evidencias científicas (Cortiñas-Rovira; Moya-Arrabal, 2018).

Uno de los problemas más urgentes que debe abordar la sociedad actual es la proliferación de pseudociencia, puesto que su elevada penetración en el tejido social y la utilización de las propias herramientas comunicativas del sistema, le confieren amplia legitimidad (Alonso-Marcos; Cortiñas-Rovira, 2014a). Por ello es imprescindible avanzar en la investigación del entorno periodístico científico mediante estudios sistemáticos (Calvo-Hernando, 2005).

Desde la visión comunicativa se propone una definición de pseudociencia como

“(1) el conjunto de prácticas que intentan apoderarse del status científico (2) mediante la exposición de unos resultados aislados, no demostrables y subjetivos, (3) obtenidos de manera acrítica, no sistemática, interesada e imprecisa, (4) para generar un conocimiento no acumulable ni válido científicamente, que (5) opera en ámbitos donde la ciencia no llega o donde no ha ofrecido resultados satisfactorios y que (6) suele ser hábil en el manejo de los medios de comunicación y de las emociones del público" (Alonso-Marcos; Cortiñas-Rovira, 2014b, p. 100).

Los medios de comunicación poseen

"la responsabilidad de velar por la imperiosa necesidad de educar e informar a la población con rigor y adhesión a los conocimientos verídicos sobre la actualidad social" (Cortiñas-Rovira; Moya-Arrabal, 2018).

Sin embargo esta condición no siempre se cumple dado que en ocasiones el relato periodístico sobre temas científicos se muestra deficiente, inadecuado, incompleto, poco preciso y carente de independencia (Ashwell, 2016).

Autores como Lavorgna y Di-Ronco (2018) sostienen que la prensa ha transmitido mensajes confusos y ambivalentes sobre las terapias alternativas y complementarias, debido entre otras razones a la falta de preparación de los periodistas sobre estos temas y a la carencia de voces de expertos y organizaciones médicas en el discurso mediático. 
Esta circunstancia enlaza con las conclusiones derivadas del estudio de Cortiñas-Rovira et al. (2015) sobre cómo los informadores científicos españoles perciben el estado actual del periodismo científico con respecto a la pseudociencia, apreciándose dos posturas:

- periodistas que analizan el riesgo potencial que este fenómeno representa para la sociedad al penetrar en los medios de comunicación;

- periodistas que perciben la pseudociencia como un entretenimiento inofensivo y con escaso impacto.

Se aprecia cómo las creencias personales del informador (Arendt, 2016) y la tendencia a recurrir al sensacionalismo como reclamo periodístico (Van-Eperen; Marincola; Strohm, 2010), podrían desvirtuar su correcta cobertura periodística.

No existen pautas editoriales concretas sobre el fenómeno pseudocientífico en medios de comunicación, principalmente a causa de la falta de formación científica de sus editores y gestores, lo que favorece el incremento de publicaciones de este tipo de contenidos (Cortiñas-Rovira et al., 2015). Resulta habitual la introducción de contenidos pseudocientíficos en la agenda mediática. La presencia de la pseudociencia en los mass media motiva que ésta sea aceptada (Ericson; Baranek; Chan, 2011), debido a la credibilidad que se les supone a los medios de comunicación (Armentia, 2002).

De este modo los medios de comunicación, especialmente si anteponen sus intereses económicos a su compromiso con la sociedad, constituyen una importante plataforma que favorece la justificación del discurso pseudocientífico (Martí-Sánchez; Roger-Monzó, 2018).

\begin{abstract}
La presencia de la pseudociencia en los mass media motiva que ésta sea aceptada debido a la credibilidad que se les supone a los medios de comunicación
\end{abstract}

La presencia de estas prácticas en prensa escrita y digital, televisión y radio también es considerable (Escribà-Sales, 2014). Respecto a la homeopatía, se ha observado que los medios de comunicación españoles informan con escaso rigor científico y sanitario, especialmente si se compara con otros países como Reino Unido (Escribà-Sales; Cortiñas-Rovira; Alonso-Marcos, 2015). La colaboración entre científicos y periodistas permitiría una mejor comprensión de aspectos científicos y de la salud por parte de estos últimos, lo que les posibilitaría elaborar contenidos claros y fiables para la sociedad (Friedman; Tanner; Rose, 2014). A su vez, establecer una firme relación comunicativa de científicos y periodistas con la población, minimizaría los riesgos que conlleva la pseudociencia (Cortiñas-Rovira; Moya-Arrabal, 2018).

La homeopatía ha sido la práctica que ha tenido mayor visibilidad en la prensa digital, especialmente por abordar el debate sobre su efectividad y sobre la necesidad de una normativa específica con un sesgo mayoritariamente negativo (Moreno-Castro; Lopera-Pareja, 2016; Mendoza-Poudereux; Cano-Orón, 2017). En España el tratamiento de la homeopatía, tanto en medios impresos como digitales, es generalmente negativo, aunque se observa el insuficiente compromiso de la prensa por acercar a la sociedad un debate crítico y riguroso (Escribà-Sales; Cortiñas-Rovira; Alonso-Marcos, 2015). Conclusiones similares se ofrecen en el estudio de Martí-Sánchez; Roger-Monzó (2018), donde se revela que la homeopatía se convierte en noticia cuando se relaciona con temas de preocupación social, como la salud y la educación.

La información sobre homeopatía de la que disponen los usuarios procede fundamentalmente de amigos y conocidos, siendo internet la segunda fuente más utilizada (Cano-Orón; Mendoza-Poudereux; Moreno-Castro, 2018). Es precisamente en internet donde se observa una importante proliferación de este tipo de contenidos, debido a la efectividad de las estrategias comunicativas y comerciales que las promocionan (López-Cantos, 2017a).

Así pues, las pseudociencias y especialmente la homeopatía, gozan de un elevado grado de legitimidad entre la población y su nivel de integración social es muy amplio por diversas razones: a la estrecha relación que la homeopatía tiene con los medios de comunicación y la publicidad (Dodds; Tseelön; Weitkamp, 2008), se suma el marco regulador condescendiente sobre productos homeopáticos existente en España (Escribà-Sales; Cortiñas-Rovira; Alonso-Marcos, 2015; Gámez, 2002). Además las presiones económicas que apoyan la difusión de prácticas pseudocientíficas a través de estudios positivos llevados a cabo por entidades productoras o distribuidoras de homeopatía (Stocking; Holstein, 2009) la han convertido en un método aceptado en la sociedad y rodeado de un aura de inocuidad y cotidianeidad.

Estas circunstancias han determinado la existencia de una intensa profesionalización en el ámbito de la homeopatía (Albert-Rodrigo, 2014) que ha derivado en la proliferación de acciones formativas especializadas (Díaz-Sáez; Moreno-Sánchez; Balmy, 2012).

"La homeopatía es, sin lugar a dudas, la pseudomedicina que ha conseguido hacerse con una mayor credibilidad entre el público gracias, sobre todo, a la laxitud científica de los colegios profesionales -han primado, en el caso de ésta y otras pseudomedicinas, los intereses asociados sobre la salud de la comunidad-, a la presión de la poderosa industria farmacéutica, a unas instituciones universitarias que, irresponsablemente, se han dejado y se dejan llevar por modas, y a unos medios de comunicación que le han hecho la publicidad gratuita” (Gámez, 2002).

Un ejemplo es la creación de la Cátedra Boiron de investigación, docencia y divulgación de la homeopatía en la Universidad de Zaragoza en 2010 con la financiación de los laboratorios Boiron. Fue la primera cátedra en España que se realizó sobre homeopatía, ocasionando gran polémica y rechazo en el seno de la comunidad académica y científica (Pérez, 2013). A partir de ese momento se realizaron diversos programas de posgrado relacionados con la homeopatía en 
universidades españolas. No fue hasta 2016 que la mayor parte de estos cursos especializados fueron clausurados, en gran medida por la polémica suscitada en sectores académicos y científicos, que se han posicionado en contra de estos estudios.

La propagación de estas prácticas en la sociedad puede ocasionar problemas de salud pública (López-Cantos, 2017b), especialmente en los casos donde se concibe como una terapia alternativa y el usuario no recibe la atención médica adecuada a tiempo (Posadzki; Alotaibi; Ernst, 2012; Mulet-Salort, 2015). En caso de que tengan presencia en los medios de comunicación, deberían abordarse desde una perspectiva crítica y con rigor científico (López-Cantos; Millán-Yeste, 2018).

\subsection{Responsabilidad y poder mediáticos}

La agenda mediática establece la capacidad de los medios para modelar las actitudes, sobre todo cuando éstas todavía no se han formado en las personas. El concepto de "establecimiento de la agenda pública" (McCombs; Evatt, 1995) se relaciona con una estrategia de los medios por la que se contribuye a establecer la naturaleza y jerarquía pública de los temas de importancia social tratados en dichos medios, mediante su circulación, difusión y discusión pública.

Durante mucho tiempo esta teoría ha sido el paradigma de las investigaciones en torno a la formación de la opinión pública. No obstante, con la llegada de internet dicha teoría ha evolucionado. La multiplicación de canales y la capacidad de los ciudadanos de seleccionar la información en los medios digitales según sus intereses, ha puesto en duda el poder de los medios de comunicación tradicionales para establecer de forma unidireccional la influencia de la agenda de los medios en la agenda pública. Dichos medios digitales adquieren cada vez más relevancia en la configuración de la opinión pública (Meraz, 2009; 2011).

En el caso de la homeopatía, si es capaz de acceder y encontrar en la prensa digital un trampolín que facilite su inserción social, puede conseguir su normalización y legitimidad y de esta manera reforzar la idea de que su efectividad es una realidad (Martí-Sánchez; Roger-Monzó, 2018).

Resultan muy interesantes las aportaciones de Alsius $(1998 ; 1999 ; 2011)$ que detalla el conjunto de cuestiones éticas de la comunicación y las agrupa en torno a cuatro principios fundamentales:

- verdad;

- justicia;

- libertad;

- solidaridad o responsabilidad social.

En este último principio se propone un subgrupo titulado 'materias de especial sensibilidad social', junto con los demás subgrupos que lo integran:

- incitación a la violencia;

- información de mal gusto;

- respeto a las creencias; alarmismo;

- violencia de género.

Estos subgrupos se sitúan bajo el principio general ético de responsabilidad social, el cual se refiere a una doble responsabilidad como parte del compromiso social: la institucional de los medios de comunicación y la individual del periodista (Alonso-Marcos; Cortiñas-Rovira, 2014a).

Ya no es suficiente con un planteamiento liberal de la responsabilidad de los medios.

"Si los medios pueden hacer un bien es porque asumen responsabilidad de hacerlo y actúan conforme a dicha responsabilidad".

Este autor extiende también la responsabilidad a los periodistas, afirmando que la neutralidad no es una opción ante algunos de los problemas graves en la sociedad (Aznar-Gómez, 2004). Señala que un periodismo atento a sus propias posibilidades de mejora será sin duda un periodismo alejado del morbo, del drama fácil, de la mera sucesión de lamentos, episodios trágicos y desastres "que tanto abundan hoy en los medios y que tan poco aportan".

La novedad de este estudio con respecto a investigaciones anteriores reside en definir posicionamiento, género periodístico, encuadre y fuentes en torno a la homeopatía, en un período de tiempo en el que de forma gradual los medios han tomado conciencia de la necesidad de abordar ese fenómeno, en parte por la controversia generada en el ámbito universitario.

\section{Metodología}

\subsection{La muestra}

El objetivo de este estudio es analizar y valorar el tratamiento que realiza la prensa digital española sobre las informaciones relativas a la homeopatía. Para ello se han escogido las ediciones digitales de los tres principales periódicos de ámbito nacional de diferente orientación ideológica: elmundo.es (20.728.000 usuarios únicos), elpais.com (20.615.000 usuarios únicos) y abc.es (13.240.000 usuarios únicos), según datos de abril de 2018 de comScore. 
Posteriormente se ha accedido a los buscadores de las webs corporativas de las ediciones digitales y se ha introducido el término "homeopat". De esa manera, además de "homeopatía", se obtenían también otros términos que comparten raíz tales como "homeopático/a" y "homeópata".

Se han recuperado 292 resultados:

- 61 de abc.es

- 161 de elpais.com

- 70 de elmundo.es.

A continuación se han examinado uno por uno y en paralelo por dos investigadores con el fin de seleccionar sólo aquellos en los que se habla con profundidad de la homeopatía. El consenso final ha sido incluir en el estudio 115 artículos (33 de abc.es, 53 de elpais.com y 29 de elmundo.es); este descenso se produce al descartarse los artículos que mencionaban únicamente el término de manera metafórica. Para recuperar resultados más precisos y evitar el ruido, se han incorporado únicamente los artículos que tratan la homeopatía literalmente.

Una vez obtenido el corpus, se ha creado una base de datos que recoge, almacena y categoriza la información relevante para el estudio. La citada ficha contiene 6 campos que permiten identificar cada noticia respecto a las demás mediante el proceso de vaciado de datos y codificación de las variables:

Tabla 1. Ficha de análisis

Fecha: en la que se publica la información

Periódico digital: elpais.com; elmundo.es o abc.es

Género periodístico: noticia, reportaje, entrevista, crónica, editorial, artículo de opinión o fotonoticia.

Encuadre: framings más frecuentes en los que se enmarcan este tipo de noticias: controversia y conflicto; riesgos y seguridad; remedios/ soluciones puntuales/legitimidad y creatividad; neutro; bienestar y wellfitness; en contra y otros.

Posicionamiento: a favor, en contra, neutral o equidistante.

Fuentes de información: si hay o no en cada una de las piezas periodísticas seleccionadas.

\subsection{Análisis de contenido}

Esta técnica permite

"formular, a partir de ciertos datos, inferencias reproducibles y válidas que puedan aplicarse a su contexto" (Krippendorf, 1990).

El investigador debe tener en cuenta las siguientes premisas:

- los datos tal y como se comunican al investigador;

- el contexto de los datos;

- la manera en que el analista considera que debe categorizar la realidad.

Se han escogido los items que permiten obtener respuestas objetivas a los temas planteados.

En la misma línea, Berelson sostiene que el análisis de contenido es

"una técnica de investigación para la descripción objetiva, sistemática y cuantitativa del contenido manifiesto de la comunicación" (Berelson, 1952).

Por tanto es necesario codificar el material. La codificación consiste en una transformación mediante reglas precisas de los datos brutos del texto para representarlos en índices numéricos o alfabéticos que permitan una descripción precisa de las características de su contenido (Andréu-Abela, 2002; Holsti, 1969).

\section{Resultados}

Los artículos obtenidos en los tres diarios digitales desde el 1 de enero de 2012 al 31 de diciembre de 2016 se presentan en la tabla 1, en la que se observa que elpais. com ha sido el diario que mayor número de informaciones ha dedicado a la homeopatía, mientras que las otras dos cabeceras presentan un resultado inferior similar.

Los tres diarios muestran una tendencia estable en número de artículos si bien es el último año, 2016, el que presenta un in-

Tabla 2. Número de artículos por cabecera

\begin{tabular}{|l|l|l|l|l|l|l|}
\hline Homeopatía & $\mathbf{2 0 1 2}$ & $\mathbf{2 0 1 3}$ & $\mathbf{2 0 1 4}$ & $\mathbf{2 0 1 5}$ & $\mathbf{2 0 1 6}$ & TOTAL \\
\hline abc.es & 5 & 5 & 6 & 3 & 14 & $\mathbf{3 3}$ \\
\hline elmundo.es & 7 & 5 & 3 & 6 & 8 & $\mathbf{2 9}$ \\
\hline elpais.com & 2 & 6 & 6 & 13 & 26 & $\mathbf{5 3}$ \\
\hline Total & 14 & 16 & 15 & 22 & 48 & 115 \\
\hline
\end{tabular}


cremento importante, sobre todo en abc.es, que pasa de 3 a 14 informaciones (467\%) y elpais.com, que duplica resultados. Como se ha señalado anteriormente, la razón del incremento de resultados en 2016 corresponde a la clausura de cursos sobre terapias alternativas en distintas universidades españolas.

Con respecto a la variable géneros periodísticos, como se observa en el gráfico 1, la noticia es el género informativo más empleado con 51 registros, seguido del artículo de opinión (30), el reportaje (17) y la entrevista (12). De estos datos se deduce que los periódicos consideran la homeopatía como hecho relevante de actualidad y los contenidos relacionados con las pseudoterapias, por formar parte del debate público, adquieren relevancia en la agenda mediática.

La taxonomía clásica de Laswell (1948) en cuanto a las funciones de los medios (vigilancia del entorno, correlación entre las partes y transmisión) se cumple en el tratamiento de la homeopatía, puesto que el segundo género presentado con más frecuencia es el opinativo, en el que los medios dan visibilidad a voces que se posicionan sobre el tema.

Respecto al enfoque de los contenidos, destaca la controversia y el conflicto que causa la utilización de la homeopatía (83 registros). Si bien el tema sobre los riesgos y la seguridad de la homeopatía se halla en segunda posición, la distancia con respecto al anterior es muy significativa (12 registros). Este dato revela que los periódicos se hacen eco de la polémica que suscita el uso de la homeopatía. Según los valores noticia definidos por Wolf (1999), la variable referida al impacto que la noticia puede causar, no es relevante en el tema que nos ocupa ya que los aspectos como los riesgos y la seguridad de la homeopatía no son abordados de manera prioritaria.

Los ciudadanos utilizan los medios de comunicación como fuente para obtener información referida a la salud. Dichos medios pueden ser considerados como 'motores' de la educación sanitaria de la población, puesto que desempeñan la función de divulgación médica (Peñafiel-Saiz et al., 2014). El rigor y la seriedad en el tratamiento informativo de los contenidos sobre salud es crucial, ya que puede aportar amplios beneficios y ser útiles para la sociedad, siempre que se evite el sensacionalismo y no se creen falsas expectativas (Blanco-Castilla; Paniagua-Rojano, 2007).

Si bien tradicionalmente se considera la objetividad periodística como axioma profesional en los medios de comunicación, en determinados temas de interés social y que pueden afectar a la salud es necesario plantearse cuestiones de éti- 
ca periodística que no existían y a los que ahora hay que dar respuesta. Esto supone que ante estas cuestiones los medios deben ser responsables y no deben mostrarse equidistantes sino posicionarse.

En este caso se comprueba un posicionamiento contrario al uso de la homeopatía, ya que el $65 \%$ de los contenidos están "en contra". Existe un 22\% de informaciones que adquieren un enfoque "neutro", y las posiciones "a favor" y "equidistante" ( $8 \%$ y $5 \%$, respectivamente), si bien son minoritarias, tienen una presencia que conviene tener en cuenta.

Si se realiza este análisis por cada uno de los medios, tal y como se aprecia en el gráfico 4, elpais.com tiene una posición "en contra" de la homeopatía en todos los contenidos, mientras que se mantiene "equidistante" en 3 registros y "neutro" en 7. En elmundo.es las posiciones "en contra" son 16, mostrándose "a favor" y "equidistante" en 2 ocasiones y "neutro" en 9 casos. Por su parte, el periódico abc.es se posiciona "en contra" en 16 ocasiones, mientras que se muestra "a favor" en 7 registros. La posición "equidistante" se da en un único registro, si bien adopta el enfoque "neutro" en 9 contenidos.

Teniendo en cuenta que en el periodismo sobre temas de salud es habitual manejar información compleja que puede generar un efecto contrario al que se pretende, es necesario que los contenidos se acompañen de referencias y consejos que redunden en el bienestar de la sociedad (Blanco-Castilla; Paniagua-Rojano, 2007). Por ello en el estudio que se presenta es de interés destacar la existencia de contenidos cuyo posicionamiento es "a favor" y "equidistante" con respecto a la homeopatía, especialmente si se tiene en cuenta que los expertos abogan por la necesidad de otorgar importancia destacada al componente educativo que este tipo de informaciones deben tener para que posean calidad informativa y de servicio público (Peñafiel-Saiz et al., 2014).

En relación con las fuentes de información, la investigación revela que en 97 registros se ha recurrido a fuentes de información para argumentar las afirmaciones que se realizan sobre la homeopatía, si bien 18 contenidos (16\%) carecen de ellas; de estos últimos, 8 pertenecen a elpais.com, 8 a elmundo.es y 2 a abc.es.

El valor de la información periodística está relacionado con la cantidad de fuentes, la calidad de las mismas y su pluralismo. Para que los contenidos sobre temas de salud sean de calidad, es imprescindible el conocimiento y cita de las fuentes, así como contrastar la información aportada por las mismas. Además, en la elaboración de este tipo de informaciones se precisa la cooperación entre profesionales de la salud y la comunicación (Peñafiel-Saiz et al., 2014).

En la investigación que se desarrolla se constata que, aunque en la mayor parte de los casos se indican fuentes de información (84\%), un $16 \%$ no contiene alusión directa a las mismas, sino que la información ha sido elaborada con fuentes propias.

\section{Conclusiones}

El papel que desempeñan los medios de comunicación en la sociedad actual es fundamental para transmitir información veraz y de calidad orientada a generar debate público y tomar decisiones para avanzar en el conocimiento sobre la salud 
y el bienestar de la población. Representan una puerta de entrada esencial para la integración de contenidos en cualquier área de la sociedad. Por ello, si los contenidos difundidos son incompletos o equívocos es probable que los usuarios tiendan a formarse expectativas distorsionadas y de escaso rigor científico que incluso pueden ser perjudiciales para la salud (Schwitzer, 2008).
La construcción de la homeopatía como relato a través de los medios analizados es mayoritariamente negativa

Este estudio longitudinal confirma el posicionamiento contrario de los diarios digitales de mayor audiencia en España en relación con la homeopatía, y asimismo está avalado por otros trabajos que obtuvieron resultados similares en estudios recientes (Escribà-Sales; Cortiñas-Rovira; Alonso-Marcos, 2015; Moreno-Castro; Lopera-Pareja, 2016; Mendoza-Poudereux; Cano-Orón, 2017; Martí-Sánchez; Roger-Monzó, 2018). Sin embargo, en este estudio hemos constatado que, a pesar de los resultados obtenidos en contra del uso de la homeopatía, desde el punto de vista ético el tratamiento ha sido enmarcado en el frame de "conflicto y controversia", frente al de "riesgos en la salud". Se infiere que los diarios anteponen lo conflictivo. En términos de género periodístico, que los contenidos se publiquen en "noticia" y no en reportajes, que tengan presencia testimonial, revela por parte de la prensa una falta de tratamiento detallado y de la necesaria reflexión sobre las consecuencias del uso de la homeopatía.

Por otra parte, se constata que el incremento del uso de la homeopatía entre la población española no está vinculado con la posición que adopta la prensa, pues la construcción de la homeopatía como relato periodístico a través de los medios analizados es mayoritariamente negativa. Los resultados están alineados con las conclusiones del estudio de Moreno-Castro; Lopera-Pareja (2016), en el que se concluye que el impacto mediático de las pseudoterapias no está relacionado con el uso y los hábitos de las mismas por parte de los ciudadanos. Por otra parte, la información sobre homeopatía de la que disponen los usuarios procede fundamentalmente de amigos y conocidos, siendo internet la segunda fuente más utilizada (Cano-Orón et al., 2018).

Las limitaciones de este trabajo se encuentran en los propios medios digitales analizados y en el período de tiempo, ya que se desconoce cómo se modificarían los resultados incluyendo otros medios digitales y en un período de tiempo más amplio. Por tanto, quedan numerosas líneas de trabajo abiertas a nuevos estudios. Se considera que algunos de ellos estarían relacionados con el compromiso ético y con las guías de buenas prácticas. Se publica y se difunde información sobre prácticas basadas en la evidencia científica y no sobre aquellas que están al margen. Por ello se proponen investigaciones en las que se compruebe el valor de los resultados obtenidos tras un análisis, para constatar si existe la validación de expertos que hayan fomentado la colaboración con los periodistas científicos (Friedman et al., 2014), con gestores y con los editores de los medios.

Los medios de comunicación que cuenten entre sus rutinas periodísticas con la validación de expertos, serán capaces de suministrar información completa que evite confusiones

\section{Referencias}

Albert-Rodrigo, María (2014). "La proliferación de las medicinas alternativas y complementarias. El ejemplo de la ciudad de Valencia”. Revista de antropología experimental, n. 14, pp. 171-188.

https://revistaselectronicas.ujaen.es/index.php/rae/article/view/1790

Alonso-Marcos, Felipe; Cortiñas-Rovira, Sergi (2014a). “La pseudociencia y el poder de los medios de comunicación. La problemática ausencia de bases teóricas para afrontar el fenómeno". Historia y comunicación social, v. 9, pp. 93-103. http://revistas.ucm.es/index.php/HICS/article/view/45111

Alonso-Marcos, Felipe; Cortiñas-Rovira, Sergi (2014b). "La pseudociencia como (des)información tóxica. Una taxonomía para comprender el fenómeno y sus manifestaciones”. Ámbitos. Revista internacional de comunicación, $1 \mathrm{r}$ trimestre, n. 24. https://institucionales.us.es/ambitos/?p=910

Alsius, Salvador (1998). Ètica i periodisme. Barcelona: Pòrtic. ISBN: 9788473064835

Alsius, Salvador (1999). Codis ètics del periodisme televisiu. Barcelona: Pòrtic. ISBN: 9788473065702

Alsius, Salvador (2011). “Cap a una gran base de dades per a l'estudi de l'ètica periodística”. Periodística, n. 13, pp. 27-57. https://www.raco.cat/index.php/Periodistica/article/view/264417

Andréu-Abela, Jaime (2002). Las técnicas de análisis de contenido: una revisión actualizada. Centro de Estudios Andaluces. http://mastor.cl/blog/wp-content/uploads/2018/02/Andreu.-analisis-de-contenido.-34-pags-pdf.pdf

Arendt, Florian (2016). "Journalists' attitudes towards homeopathy: Survey data from Germany". Focus on alternative and complementary therapies, v. 21, n. 1, pp. 17-21.

https://doi.org/10.1111/fct.12244

Armentia, Javier (2002). “Ciencia vs pseudociencias”. Mediatika. Cuadernos de medios de comunicación, v. 8, pp. $559-571$. http://ojs.eusko-ikaskuntza.eus/index.php/mediatika/article/view/127/0 
Ashwell, Douglas-James (2016). "The challenges of science journalism: The perspectives of scientists, science communication advisors and journalists from New Zealand". Public understanding of science, v. 25, n. 3, pp. 379-392.

https://doi.org/10.1177/0963662514556144

Aznar-Gómez, Hugo (2004). "Nuevos códigos de ética y nuevas formas de entender el periodismo". Revista latina de comunicación social, n. 58.

http://www.revistalatinacs.org/20042158aznar.htm

Benkemoun, Pierre; Cornillot, Pierre; Deltombre-Kopp, Micheline; Lützler, Louis-Joseph; Poitevin, Bernard; Sarembaud, Alain; Solon, Michel (2002). Tratado de homeopatía. Barcelona: Editorial Paidotribo. ISBN: 9788480194402

Berelson, Bernard (1952). Content analysis in comunication research. New York: Free Press, Glencoe. ISBN: 9780 028412108

Blanco-Castilla, Elena; Paniagua-Rojano, Francisco-Javier (2007). “Periodismo, salud y calidad de vida. Contenidos y fuentes". Fisec- Estrategias, año III, n. 8, mesa II, pp. 3-24.

https://www.researchgate.net/publication/240631218_PERIODISMO_SALUD_Y_CALIDAD_DE_VIDA_CONTENIDOS_Y_FUENTES

Bracanović, Tomislav (2012). “From integrative bioethics to pseudosciencie”. Developing world bioethics, v. 12, n. 3, pp. 148-156.

https://doi.org/10.1111/j.1471-8847.2012.00330.x

Calvo-Hernando, Manuel (2005). Periodismo científico y divulgación de la ciencia. Madrid: Acta y Cedro.

Cano-Orón, Lorena; Mendoza-Poudereux, Isabel; Moreno-Castro, Carolina (2018). "Perfil sociodemográfico del usuario de la homeopatía en España". Atención primaria, pp. 1-7.

https://doi.org/10.1016/j.aprim.2018.07.006

CIS (2018). Barómetro de febrero. Estudio 3205. Madrid: Centro de Investigaciones Sociológicas.

http://www.cis.es/cis/export/sites/default/-Archivos/Marginales/3200_3219/3205/es3205mar.pdf

Cornejo-Valle, Mónica; Blázquez-Rodríguez, Maribel (2013). “La convergencia de salud y espiritualidad en la sociedad postsecular. Las terapias alternativas y la constitución del ambiente holístico”. Revista de antropología experimental, v. 13, pp. 11-30.

http://revista.ujaen.es/huesped/rae/articulos2013/02cornejo13.pdf

Cortiñas-Rovira, Sergi; Alonso-Marcos, Felipe; Pont-Sorribes, Carles; Escribà-Sales, Eudald (2015). "Science journalists' perceptions and attitudes to pseudoscience in Spain". Public understanding of science, v. 24, n. 4, pp. 450-465. https://doi.org/10.1177/0963662514558991

Cortiñas-Rovira, Sergi; Moya-Arrabal, Miguel-Ángel (2018). “La falsa-ciència (pseudociència) als mitjans de comunicació: estudi de les estratègies discursives d'inserció social a la premsa espanyola (2011-2016)". Communication papers. Media literacy \& gender studies, v. 7, n. 13, pp. 129-144.

https://dialnet.unirioja.es/servlet/articulo?codigo $=6442317$

Díaz-Sáez, Gualberto; Moreno-Sánchez, Gemma; Balmy, Sylvaine (2012): "Estudio sobre conocimiento y uso de la homeopatía en España". Revista médica de homeopatía, v. 5, n. 3, pp. 113-119.

http://supersalud.org/wp-content/uploads/2013/12/Estudio-sobre-conocimiento-y-uso-de-homeopati\%cC\%81a-enEspan\%CC\%83a.pdf

https://doi.org/10.1016/S1888-8526(12)70157-1

Dodds, Rachel E.; Tseelön, Efrat; Weitkamp, Emma L. C. (2008). "Making sense of scientific claims in advertising. A study of scientifically aware consumers". Public understanding of science, v. 17, n. 2, pp. 211-230.

https://doi.org/10.1177/0963662506065559

Ericson, Richard-Victor; Baranek, Patricia M.; Chan, Janet-Bick-Lai (2011). "Negotiating control. A study of news sources". Toronto: University of Toronto Press. ISBN: 9780802066916

Ernst, Edzard (2008). Healing. Hype or harm? A critical analysis of complementary or alternative medicine. Exeter: Societas, Imprint Academic. ISBN: 9781845401184

Ernst, Edzard (2009). "Winnowing the chaff of charlatanism from the wheat of science". Evidence-based complementary and alternative medicine, v.7, n. 4, pp. 425-426.

https://doi.org/10.1093/ecam/nen089

Escribà-Sales, Eudald (2014). “La convivència del rigor i la llibertat d'expressió a 'La contra' de La vanguardia. Estudi de cas de les entrevistes amb contingut pseudocientífic”. Comunicació. Revista de recerca i d'anàlisi, v. 31, n. 2, pp. $71-91$. https://www.raco.cat/index.php/Comunicacio/article/view/286446 
Escribà-Sales, Eudald; Cortiñas-Rovira, Sergi; Alonso-Marcos, Felipe (2015). "La pseudociencia en los medios de comunicación: estudio de caso del tratamiento de la homeopatía en la prensa española y británica durante el período 20092014". Panace@, v. 16, n. 42, pp. 177-183.

https://dialnet.unirioja.es/servlet/articulo?codigo=5350796

Fecyt (2017). VIII Encuesta de percepción social de la ciencia y la tecnología. Informe de resultados 2016. Madrid: Fundación Española para la Ciencia y Tecnología.

https://www.fecyt.es/sites/default/files/news/attachments/2017/04/epscyt2016_informe_final_web_fecyt.pdf

Friedman, Daniela B.; Tanner, Andrea; Rose, India D. (2014). “Health journalists' perceptions of their communities and implications for the delivery of health information in the news". Journal of community health, v. 39, n. 2, pp. 378-385.

https://doi.org/10.1007/s10900-013-9774-x

Gámez, Luis-Alfonso (2002). “Los periodistas y las falsas ciencias”. Mediatika: cuadernos de medios de comunicación, v. 8, pp. 27-37.

https://core.ac.uk/download/pdf/11497785.pdf

Holsti, Ole R. (1969). Content analysis for the social sciences and humanities. Massachusetts: Addison-Wesley. ISBN: 978 0394349268

Krippendorf, Klaus (1990). Metodología de análisis de contenido. Teoría y práctica. Barcelona: Paidós. ISBN: 97884 75096278

Kurtz, Paul (1978). "Is parapsychology a science?". The skeptical inquirer, v. 3, n. 2, pp. 14-23.

Lasswell, Harold D. (1948). The structure and function of communication in society. Chicago: Chicago University Press.

Lavorgna, Anita; Di-Ronco, Anna (2018). "Media representations of complementary and alternative medicine in the Italian press: A criminological perspective". European journal of criminology, v. 15, n. 4, pp. 421-441.

https://doi.org/10.1177/1477370817748589

Leaf, Justin B.; Kassardjian, Aline; Oppenheim-Leaf, Misty L.; Cihon, Joseph H.; Taubman, Mitchell; Leaf, Ronald; McEachin, John (2016). "Social thinking ${ }^{\circledR}:$ Science, pseudoscience, or antiscience?". Behavior analysis in practice, v. 9, n. 2, pp. 152-157.

https://doi.org/10.1007/s40617-016-0108-1

López-Cantos, Francisco (2017a). “Comunicación pública de la pseudociencia: homeópatas y orgonitas 2.0”. Razón y palabra, v. 21, n. 1_96, pp. 355-372.

http://revistarazonypalabra.org/index.php/ryp/article/view/836

López-Cantos, Francisco (2017b). "El discurso de la felicidad de las terapias alternativas en Facebook". Razón y palabra, v. 21, n. 3_98, pp. 381-393.

http://www.revistarazonypalabra.org/index.php/ryp/article/view/953

López-Cantos, Francisco; Millán-Yeste, J. (2018). “La difusión de discursos pseudocientíficos en la radio pública española. El programa Complementarios de RNE-Radio 5”. Revista latina de comunicación social, n. 73, pp. 317-330.

https://doi.org/10.4185/RLCS-2018-1257

Martí-Sánchez, Myriam; Roger-Monzó, Vanessa (2018). “La percepción social de la homeopatía en la prensa digital española: un análisis semántico". Panace@, v. 18, n. 47, pp. 115-123.

https://dialnet.unirioja.es/servlet/articulo?codigo $=6629817$

Mathie, Robert T.; Ramparsad, Nitish; Legg, Lynn A.; Clausen, Jürgen; Moss, Sian; Davidson, Jonathan R. T.; Messou, Claudina-Martina; McConnachie, Alex (2017). "Randomised, double-blind, placebo-controlled trials of non-individualised homeopathic treatment: Systematic review and meta-analysis". Systematic reviews, v. 6, n. 1, pp. 63.

https://doi.org/10.1186/s13643-017-0445-3

McCombs, Maxwell; Evatt, Dixie (1995). “Los temas y los aspectos: explorando una nueva dimensión de la agenda setting". Comunicación y sociedad, v. 8, n. 1, pp. 7-32.

https://www.unav.es/fcom/communication-society/es/articulo.php?art_id=169

Mendoza-Poudereux, Isabel; Cano-Orón, Lorena (2017). “El boom informativo de la homeopatía. Estudio longitudinal

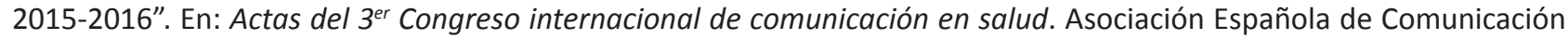
Sanitaria (AECS). Universidad Carlos III de Madrid, 19-20 de octubre.

https://e-archivo.uc3m.es/handle/10016/25575

Meraz, Sharon (2009). "Is there an elite hold? Traditional media to social media agenda setting influence in blog networks". Journal of computer-mediated communication, v. 14, n. 3, pp. 682-707.

https://doi.org/10.1111/j.1083-6101.2009.01458.x 
Meraz, Sharon (2011). "The fight for 'how to think': Traditional media, social networks, and issue interpretation". Journalism, v. 12, n. 1, pp. 107-127.

https://doi.org/10.1177/1464884910385193

Ministerio de Sanidad (2011). Análisis de la situación de las terapias naturales. Ministerio de Sanidad, Política Social e Igualdad.

http://www.mspsi.gob.es/novedades/docs/analisisSituacionTNatu.pdf

Moreno-Castro, Carolina; Lopera-Pareja, Emilia H. (2016). "Comparative study of the frequency of use of natural therapies among the Spanish population and their public image on digital media". En: Proceedings of the $14^{\text {th }}$ Int $\mathrm{conf}$ on public communication of science and technology (PCST). Estambul, Turquía, 26-28 de abril.

https://pcst.co/archive/paper/2623

Mulet-Salort, José-Miguel (2015). Medicinas sin engaños. Todo lo que necesitas saber sobre los peligros de la medicina alternativa. Barcelona: Ediciones Destino. ISBN: 9788423349043

Peñafiel-Saiz, Carmen; Camacho-Markina, Idoia; Aiestaran-Yarza, Alazne; Ronco-López, Milagros; Echegaray-Eizaguirre, Lázaro (2014). "La divulgación de la información de salud: un reto de confianza entre sectores implicados". Revista latina de comunicación social, n. 69, pp. 135-151.

http://www.revistalatinacs.org/069/paper/1005_UPV/08c.html

Pérez, Carlos-Elías (2013). “Contraconocimiento y pandemias de credulidad en la sociedad red: el papel del periodismo en la búsqueda de la verdad en los entornos digitales”. Estudios sobre el mensaje periodístico, n. 19, pp. 667-681.

http://revistas.ucm.es/index.php/ESMP/article/view/43465

Posadzki, Paul; Alotaibi, Abdulellah; Ernst, Edzard (2012). "Adverse effects of homeopathy: a systematic review of published case reports and case series". International journal of clinical practice, v. 66, n. 12, pp. 1178-1188.

https://doi.org/10.1111/ijcp.12026

Rutten, A. Lex B.; Stolper, Christiaan F. Erik (2009). "Reply to Wilson". Homeopathy, v. 98, n. 2, p. 129. https://doi.org/10.1016/j.homp.2009.01.002

Schwitzer, Gary (2008). "How do US journalists cover treatments, tests, products, and procedures? An evaluation of 500 stories". PLoS med, v. 5, n. 5, pp. 700-704.

https://doi.org/10.1371/journal.pmed.0050095

Stocking, S. Holly; Holstein, Lisa W. (2009). "Manufacturing doubt: journalists' roles and the construction of ignorance in a scientific controversy". Public understanding of science, v. 18, n. 1, pp. 23-42.

https://doi.org/10.1177/0963662507079373

Van-Eperen, Laura; Marincola, Franceso M.; Strohm, Jennifer (2010). "Bridging the divide between science and journlism". Journal of translational medicine, v. 8, n. 25, pp. 1-3.

https://doi.org/10.1186/1479-5876-8-25

Wolf, Mauro (1987). La investigación de la comunicación de masas. Críticas y perspectivas. Barcelona, Paidós. ISBN: 84 75094376

https://catedracoi2.files.wordpress.com/2014/05/wolf-mauro-investigacion-de-la-comunicacion-de-masas.pdf

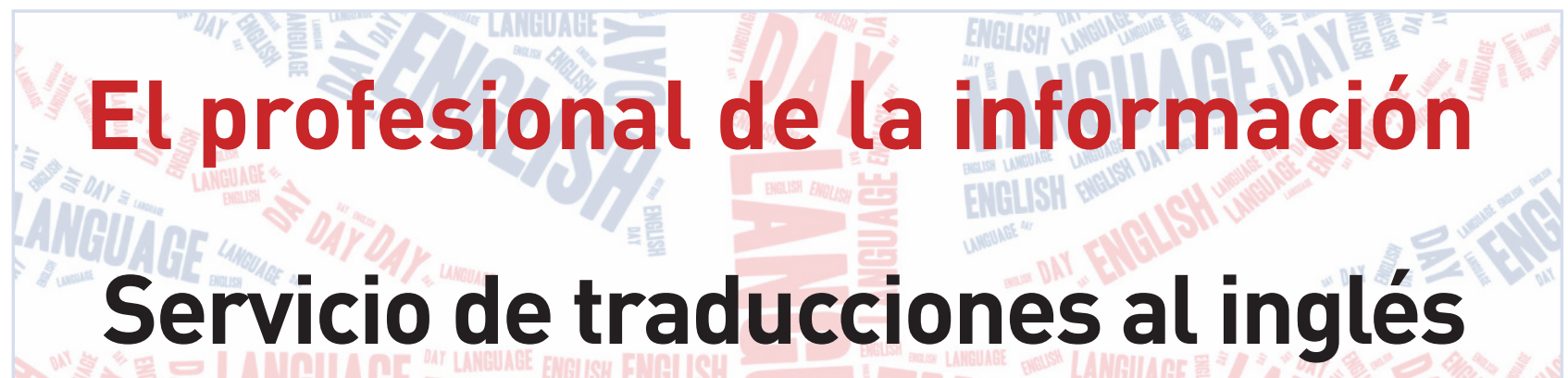

http://WwW.elprofesionaldelainformacion.com/documentos/traducciones.pdf

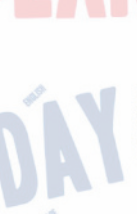

Información: Isabel Olea

epi.ioleadgmail.com 